\title{
Classification of Continuous Fractional Binary Operations on the Real and Complex Fields
}

\author{
Sin-Ei TAKAHASI, Makoto TSUKADA and Yuji KOBAYASHI \\ Toho University \\ (Communicated by K. Takemura)
}

\begin{abstract}
In this paper, we consider a classification problem for continuous fractional binary operations on $\mathbf{K}$, where $\mathbf{K}$ denotes the real field $\mathbf{R}$ or the complex field $\mathbf{C}$. We first show that there exist exactly two continuous fractional binary operations on $\mathbf{R}$ up to isomorphism. In the complex case, we describe completely all continuous fractional binary operations on $\mathbf{C}$ in terms of ordinary fraction. Applying this description, we give a partial solution to the classification problem in the complex case. Moreover we show that there exist exactly two homogeneous cancellative binary operations on $\mathbf{K}$ up to isomorphism.
\end{abstract}

\section{Introduction}

Recently S. Saitoh gave the formal identities $100 / 0=0$ and $0 / 0=0$ by the concept of Tikhonov regularization using the theory of reproducing kernels. Also he asked whether there exist some real examples supporting the above results (cf. [1, 2]). Actually take two real numbers $a, b$ arbitrarily. For any positive number $t$,

$$
x_{t}=\frac{a b}{t+b^{2}}
$$

is a value which minimizes the Tikhonov function $t x^{2}+(b x-a)^{2}$. This is called the fractional in the sense of Tikhonov. Put

$$
S(a, b)=\lim _{t \rightarrow+0} x_{t}
$$

Then we have

$$
S(a, b)= \begin{cases}a / b & (b \neq 0), \\ 0 & (b=0) .\end{cases}
$$

We call $S(a, b)$ Saitoh's fraction. Of course we can consider Saitoh's fraction in the complex case.

Received March 27, 2014; revised August 28, 2014

2000 Mathematics Subject Classification: 42A38, 30 C40 (Primary), 46E22, 32A36 (Secondary)

Key words and phrases: Tikhonov regularization, fraction, binary operation 
In this paper, inspired by his idea, we investigate the continuous fractional binary operations on $\mathbf{K}$ (see the next section for the definition). Here $\mathbf{K}$ denotes the field $\mathbf{R}$ of real numbers or the field $\mathbf{C}$ of complex numbers.

In fact our purpose is to classify all continuous fractional binary operations on $\mathbf{K}$. We first show that there exist exactly two continuous fractional binary operations on $\mathbf{R}$ up to isomorphism (see Theorem 1). In the complex case, we completely describe all continuous fractional binary operations on $\mathbf{C}$ in terms of ordinary fraction (see Theorem 2). Applying this description, we give a partial solution to the classification problem in the complex case (see Theorems 3 and 4). Moreover we show that there exist exactly two homogeneous cancellative binary operations on $\mathbf{K}$ up to isomorphism (see Theorem 5).

\section{Preliminary and main results}

Let $*$ be a binary operation on $\mathbf{K}$. We say that $*$ is fractional if

$$
(a+b) * c=(a * c)+(b * c) \text { (distribution) }
$$

and

$$
(a x) *(b x)=a * b \text { (cancellation) }
$$

for all $a, b, c, x \in \mathbf{K}$ with $x \neq 0$. Also we say that $*$ is continuous if the map : $x \mapsto x * b$ is continuous on $\mathbf{K}$ for each $b \in \mathbf{K}$. Moreover we say that $*$ is homogeneous if

$$
(a b) * c=a(b * c)
$$

for all $a, b, c \in \mathbf{K}$. Of course, the binary operation $*$ on $\mathbf{K}$ defined by $a * b=0(a, b \in \mathbf{K})$ is continuous, fractional and homogeneous. Such a binary operation is said to be trivial. Let $\mathcal{C F}(\mathbf{K})$ be the set of all continuous fractional binary operations on $\mathbf{K}$. For two operations $*, \circ \in \mathcal{C F}(\mathbf{K})$ we say that $*$ is isomorphic to $\circ$ (simply $* \cong 0$ ) if there exists a homeomorphism $f: \mathbf{K} \rightarrow \mathbf{K}$ such that

$$
f(a * b)=f(a) \circ f(b)
$$

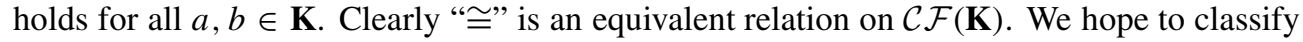
all continuous fractional binary operations on $\mathbf{K}$ modulo " $\cong$ ".

The first classification result is the following theorem which asserts that there exist exactly two continuous fractional binary operations on $\mathbf{R}$ up to isomorphism.

THEOREM 1. All nontrivial continuous fractional binary operations on $\mathbf{R}$ are isomorphic to Saitoh's fraction on $\mathbf{R}$.

For the complex case, we can completely describe all continuous fractional binary operations on $\mathbf{C}$ in terms of ordinary fraction as follows: 
THEOREM 2. If $*$ is a continuous fractional binary operation on $\mathbf{C}$, then there exist two unique complex numbers $\alpha$ and $\beta$ such that

$$
z * w= \begin{cases}\alpha \operatorname{Re} \frac{z}{w}+i \beta \operatorname{Im} \frac{z}{w} & (w \neq 0) \\ 0 & (w=0),\end{cases}
$$

for all $z, w \in \mathbf{C}$. Conversely, the binary operation given by (1) is a continuous fractional binary operation on $\mathbf{C}$.

We denote by $*_{(\alpha, \beta)}$ the binary operation defined by (1). Then the map

$$
\Phi:(\alpha, \beta) \mapsto *(\alpha, \beta)
$$

is a bijection from $\mathbf{C}^{2}$ to $\mathcal{C F}(\mathbf{C})$.

REMARK. $\Phi(0,0)$ is the trivial fractional binary operation on C. Also $\Phi(1,1)$ is just Saitoh's fraction on $\mathbf{C}$.

Let $\hat{\mathbf{C}}=\mathbf{C} \cup\{\infty\}$ and put

$$
L_{\gamma}= \begin{cases}\left\{(\alpha, \beta) \in \mathbf{C}^{2} \backslash\{(0,0)\}: \beta=\alpha \gamma\right\} & (\gamma \in \mathbf{C}) \\ \left\{(\alpha, \beta) \in \mathbf{C}^{2} \backslash\{(0,0)\}: \alpha=0\right\} & (\gamma=\infty) .\end{cases}
$$

Then we have

$$
\mathbf{C}^{2}=\{(0,0)\} \cup \bigcup_{\gamma \in \hat{\mathbf{C}}} L_{\gamma} \quad \text { (disjoint union) } .
$$

The following two theorems give a partial solution to the classification problem in the complex case.

THEOREM 3. For each $\gamma \in \hat{\mathbf{C}}$, it holds that $\Phi(\alpha, \beta) \cong \Phi\left(\alpha^{\prime}, \beta^{\prime}\right)$ for all $(\alpha, \beta),\left(\alpha^{\prime}, \beta^{\prime}\right) \in L_{\gamma}$.

THEOREM 4. (i) $\Phi(0,0)$ is not isomorphic to any nontrivial continuous fractional binary operation on $\mathbf{C}$.

(ii) If $\gamma=0$ or $\infty$ and $\gamma^{\prime} \in \mathbf{C} \backslash\{0\}$, then $\Phi(\alpha, \beta) ¥ \Phi\left(\alpha^{\prime}, \beta^{\prime}\right)$ for each $(\alpha, \beta) \in L_{\gamma}$ and $\left(\alpha^{\prime}, \beta^{\prime}\right) \in L_{\gamma^{\prime}}$ with $\operatorname{Re} \alpha^{\prime} \overline{\beta^{\prime}} \neq 0$.

(iii) $\Phi(\alpha, \beta) \supsetneqq \Phi\left(\alpha^{\prime}, \beta^{\prime}\right)$ for each $(\alpha, \beta) \in L_{0}$ and $\left(\alpha^{\prime}, \beta^{\prime}\right) \in L_{\infty}$.

(iv) Let $\alpha, \beta \in \mathbf{C} \backslash\{0\}$. Then $\Phi(\alpha, \beta) \cong \Phi(1,1)$ if and only if $\alpha=\beta$.

REMARK. (a) By (ii) and (iv), we have that if $\gamma \in \hat{\mathbf{C}} \backslash\{1\}$, then $\Phi(\alpha, \beta) ¥ \Phi\left(\alpha^{\prime}, \beta^{\prime}\right)$ for each $(\alpha, \beta) \in L_{1}$ and $\left(\alpha^{\prime}, \beta^{\prime}\right) \in L_{\gamma}$. However we do not know whether $\Phi(\alpha, \beta) \cong$ $\Phi\left(\alpha^{\prime}, \beta^{\prime}\right)$ or not for each $(\alpha, \beta) \in L_{\gamma}$ and $\left(\alpha^{\prime}, \beta^{\prime}\right) \in L_{\gamma^{\prime}}$ when $\gamma, \gamma^{\prime} \in \hat{\mathbf{C}} \backslash\{1\}$ and $\gamma \neq \gamma^{\prime}$.

(b) If the function $x \mapsto 1 * x$ is continuous at $x=0$, then $*$ is trivial.

The last classification result is the following theorem which asserts that there exist exactly two homogeneous cancellative binary operations on $\mathbf{K}$ up to isomorphism. 
THEOREM 5. All nontrivial homogeneous cancellative binary operations on $\mathbf{K}$ are isomorphic to Saitoh's fraction on $\mathbf{K}$.

\section{Proof of main results}

I. Proof of Theorem 1 . Let $*$ be a nontrivial continuous fractional binary operation on $\mathbf{R}$. For each $b \in \mathbf{R}$, put

$$
R(x)=x * b \quad(x \in \mathbf{R}) .
$$

Since $R$ is a continuous additive map from $\mathbf{R}$ to itself, we can find a unique real number $\varphi(b)$ such that $R(x)=x \varphi(b)$ for all $x \in \mathbf{R}$. Therefore we have

$$
a * b=a \varphi(b)
$$

for all $a, b \in \mathbf{R}$. So $*$ is necessarily homogeneous. Take $a, b \in \mathbf{R}$ with $b \neq 0$ arbitrarily. Put $e=1 * 1$. Since $*$ is cancellative, it follows that

$$
a * b=a \varphi(b)=\frac{a b \varphi(b)}{b}=\frac{a(b * b)}{b}=\frac{a(1 * 1)}{b}=\frac{a e}{b} .
$$

Also since $*$ is cancellative and homogeneous, it follows that

$$
a * 0=(2 a) * 0=2(a * 0),
$$

and hence $a * 0=0$. Therefore we have

$$
a * b= \begin{cases}\frac{a e}{b} & (b \neq 0) \\ 0 & (b=0) .\end{cases}
$$

Since $*$ is nontrivial, it follows that $e \neq 0$. Define

$$
f(x)=\frac{x}{e}
$$

for each $x \in \mathbf{R}$. Then $f$ is a homeomorphism from $\mathbf{R}$ to itself. Take $a, b \in \mathbf{R}$ arbitrarily. If $b \neq 0$, then $f(b) \neq 0$, and hence

$$
f(a * b)=f\left(\frac{a e}{b}\right)=\frac{1}{e} \frac{a e}{b}=\frac{a}{b}=\frac{a / e}{b / e}=S(f(a), f(b)) .
$$

If $b=0$, then

$$
f(a * b)=f(0)=0=S(f(a), 0)=S(f(a), f(0))=S(f(a), f(b)) .
$$

Consequently, $*$ is isomorphic to Saitoh's fraction on $\mathbf{R}$.

II. Proof of Theorem 2. We need the following lemma. It seems that this lemma is a known result, but we give a proof for the sake of completeness. 
LEMMA 1. If $\varphi$ is a continuous additive map from $\mathbf{C}$ to itself, then it is mixed-linear, that is, there exist two unique complex numbers $\alpha$ and $\beta$ such that $\varphi(z)=\alpha z+\beta \bar{z}$ for all $z \in \mathbf{C}$.

PROOF. Let $\varphi$ be a continuous additive map from $\mathbf{C}$ to itself. Put

$$
u(x)=\operatorname{Re} \varphi(x) \text { and } v(x)=\operatorname{Im} \varphi(x)
$$

for each $x \in \mathbf{R}$. Then

$$
\varphi(x+y)=u(x+y)+i v(x+y)
$$

and

$$
\begin{aligned}
\varphi(x)+\varphi(y) & =u(x)+i v(x)+u(y)+i v(y) \\
& =u(x)+u(y)+i(v(x)+v(y))
\end{aligned}
$$

for all $x, y \in \mathbf{R}$. Since $\varphi(x+y)=\varphi(x)+\varphi(y)(x, y \in \mathbf{R})$, it follows that

$$
u(x+y)=u(x)+u(y) \text { and } v(x+y)=v(x)+v(y)
$$

hold for all $x, y \in \mathbf{R}$. Then both $u$ and $v$ are continuous additive real-valued functions on R. This implies easily that $u(x)=a x$ and $v(x)=b x$ for all real numbers $x$ and some real numbers $a, b$. We next put

$$
u^{\prime}(x)=\operatorname{Re} \varphi(i x) \quad \text { and } \quad v^{\prime}(x)=\operatorname{Im} \varphi(i x)
$$

for each $x \in \mathbf{R}$. Then

$$
\varphi(i(x+y))=u^{\prime}(x+y)+i v^{\prime}(x+y)
$$

and

$$
\begin{aligned}
\varphi(i x)+\varphi(i y) & =u^{\prime}(x)+i v^{\prime}(x)+u^{\prime}(y)+i v^{\prime}(y) \\
& =u^{\prime}(x)+u^{\prime}(y)+i\left(v^{\prime}(x)+v^{\prime}(y)\right)
\end{aligned}
$$

for all $x, y \in \mathbf{R}$. Since $\varphi(i(x+y))=\varphi(i x)+\varphi(i y)(x, y \in \mathbf{R})$, it follows that

$$
u^{\prime}(x+y)=u^{\prime}(x)+u^{\prime}(y) \text { and } v^{\prime}(x+y)=v^{\prime}(x)+v^{\prime}(y)
$$

hold for all $x, y \in \mathbf{R}$. Then both $u^{\prime}$ and $v^{\prime}$ are also continuous additive real-valued functions on $\mathbf{R}$. This implies easily that $u^{\prime}(x)=c x$ and $v^{\prime}(x)=d x$ for all real numbers $x$ and some real numbers $c, d$. Therefore

$$
\begin{aligned}
\varphi(z) & =\varphi(x+i y) \\
& =\varphi(x)+\varphi(i y) \\
& =a x+i b x+c y+i d y \\
& =(a+i b) \operatorname{Re} z+(c+i d) \operatorname{Im} z
\end{aligned}
$$


holds for all $z=x+i y \in \mathbf{C}$. Put

$$
\alpha=\frac{a+i b}{2}+\frac{c+i d}{2 i} \text { and } \beta=\frac{a+i b}{2}-\frac{c+i d}{2 i} .
$$

Then we have from the above equation that $\varphi(z)=\alpha z+\beta \bar{z}$ for all $z \in \mathbf{C}$. Moreover it will be clear that such $\alpha$ and $\beta$ are unique.

Let $*$ be a continuous fractional binary operation on $\mathbf{C}$. For each $w \in \mathbf{C}$, put

$$
f(z)=z * w \quad(z \in \mathbf{C}) .
$$

Since $f$ is a continuous additive map from $\mathbf{C}$ to itself, it follows from Lemma 1 that there exist two unique complex numbers $\varphi(w)$ and $\psi(w)$ such that $f(z)=z \varphi(w)+\bar{z} \psi(w)$ for all $z \in \mathbf{C}$. Therefore

$$
z * w=z \varphi(w)+\bar{z} \psi(w)
$$

holds for all $z, w \in \mathbf{C}$. Hence we have

$$
(r z) * w=r(z * w)
$$

for all $r \in \mathbf{R}$ and $z, w \in \mathbf{C}$. Put

$$
\alpha=1 * 1 \quad \text { and } \quad \beta=\frac{i * 1}{i} .
$$

If $x$ is a nonzero real number, we have from (2) that

$$
\alpha=1 * 1=x * x=x \varphi(x)+\bar{x} \psi(x)=x(\varphi(x)+\psi(x))
$$

holds because $*$ is cancellative. Then

$$
\varphi(x)+\psi(x)=\frac{\alpha}{x}
$$

holds for all $x \in \mathbf{R} \backslash\{0\}$. Similarly we have that if $x$ is a nonzero real number, then

$$
i \beta=i * 1=(x i) * x=x i \varphi(x)+\overline{x i} \psi(x)=x i \varphi(x)-x i \psi(x)
$$

holds. Then

$$
\varphi(x)-\psi(x)=\frac{\beta}{x}
$$

holds for all $x \in \mathbf{R} \backslash\{0\}$. Therefore we have from (4) and (5) that

$$
\left\{\begin{array}{l}
\varphi(x)=\frac{\alpha+\beta}{2 x} \\
\psi(x)=\frac{\alpha-\beta}{2 x}
\end{array}\right.
$$

holds for all $x \in \mathbf{R} \backslash\{0\}$. Then we have from (2) and (6) that

$$
x * y=x \varphi(y)+x \psi(y)=\frac{x(\alpha+\beta)}{2 y}+\frac{x(\alpha-\beta)}{2 y}=\frac{x \alpha}{y}
$$


holds for all $x, y \in \mathbf{R}$ with $y \neq 0$. Note that

$$
i * x=\frac{i}{x} * \frac{x}{x}=\frac{i}{x} * 1=\frac{1}{x}(i * 1)=\frac{i \beta}{x}
$$

holds for all $x \in \mathbf{R} \backslash\{0\}$. If $a, b \in \mathbf{R}$ with $a^{2}+b^{2} \neq 0$, then we have from (3), (7) and (8) that

$$
\begin{aligned}
i *(a+i b) & =((a-i b) i) *\left(a^{2}+b^{2}\right) \\
& =(b+i a) *\left(a^{2}+b^{2}\right) \\
& =b *\left(a^{2}+b^{2}\right)+(a i) *\left(a^{2}+b^{2}\right) \\
& =\frac{b \alpha}{a^{2}+b^{2}}+\frac{a i \beta}{a^{2}+b^{2}} \\
& =\frac{b \alpha+a i \beta}{a^{2}+b^{2}}
\end{aligned}
$$

and

$$
\begin{aligned}
1 *(a+i b) & =(a-i b) *\left(a^{2}+b^{2}\right) \\
& =a *\left(a^{2}+b^{2}\right)-b\left(i *\left(a^{2}+b^{2}\right)\right) \\
& =\frac{a \alpha}{a^{2}+b^{2}}-\frac{b i \beta}{a^{2}+b^{2}} \\
& =\frac{a \alpha-b i \beta}{a^{2}+b^{2}} .
\end{aligned}
$$

Therefore if $z=a+i b, w=c+i d \neq 0$, then

$$
\begin{aligned}
z * w & =a(1 * w)+b(i * w) \\
& =\frac{a(c \alpha-d i \beta)}{c^{2}+d^{2}}+\frac{b(d \alpha+c i \beta)}{c^{2}+d^{2}} \\
& =\frac{(a c+b d) \alpha+(b c-a d) i \beta}{c^{2}+d^{2}} \\
& =\frac{\alpha \operatorname{Re}(z \bar{w})+i \beta \operatorname{Im}(z \bar{w})}{|w|^{2}} \\
& =\alpha \operatorname{Re}\left(\frac{z \bar{w}}{|w|^{2}}\right)+i \beta \operatorname{Im}\left(\frac{z \bar{w}}{|w|^{2}}\right) \\
& =\alpha \operatorname{Re}\left(\frac{z}{w}\right)+i \beta \operatorname{Im}\left(\frac{z}{w}\right) .
\end{aligned}
$$

Moreover since $*$ is cancellative, it follows from (3) that

$$
z * 0=0
$$


holds for all $z \in \mathbf{C}$ as observed in the proof of Theorem 1. Hence we have

$$
z * w= \begin{cases}\alpha \operatorname{Re}\left(\frac{z}{w}\right)+i \beta \operatorname{Im}\left(\frac{z}{w}\right) & (w \neq 0) \\ 0 & (w=0)\end{cases}
$$

To show the uniqueness of $\alpha$ and $\beta$, suppose that

$$
\alpha \operatorname{Re}\left(\frac{z}{w}\right)+i \beta \operatorname{Im}\left(\frac{z}{w}\right)=\alpha^{\prime} \operatorname{Re}\left(\frac{z}{w}\right)+i \beta^{\prime} \operatorname{Im}\left(\frac{z}{w}\right)
$$

holds for all $z, w \in \mathbf{C}$ with $w \neq 0$. Taking $z=w=1$ in the above equation, we have $\alpha=\alpha^{\prime}$. Also taking $z=i$ and $w=1$ in the above equation, we have $i \beta=i \beta^{\prime}$ and hence $\beta=\beta^{\prime}$. Then $\alpha$ and $\beta$ are unique.

III. Proof of Theorem 3. Let $\gamma \in \hat{\mathbf{C}}$ and $(\alpha, \beta),\left(\alpha^{\prime}, \beta^{\prime}\right) \in L_{\gamma}$. Then we must show that $\Phi(\alpha, \beta) \cong \Phi\left(\alpha^{\prime}, \beta^{\prime}\right)$. Note that $\beta=\alpha \gamma$ and $\beta^{\prime}=\alpha^{\prime} \gamma$.

(a) The case where $\gamma \in \mathbf{C} \backslash\{0\}$. Note that $\alpha \neq 0, \beta \neq 0, \alpha^{\prime} \neq 0$ and $\beta^{\prime} \neq 0$. Then we have

$$
\frac{\beta}{\alpha}=\frac{\beta^{\prime}}{\alpha^{\prime}}
$$

Put

$$
\lambda=\frac{\alpha^{\prime}}{\alpha}=\frac{\beta^{\prime}}{\beta}
$$

and

$$
f(z)=\lambda z
$$

for each $z \in \mathbf{C}$. Then $f$ is a homeomorphism from $\mathbf{C}$ to itself and

$$
\begin{aligned}
f(z *(\alpha, \beta) w) & =\lambda\left(\alpha \operatorname{Re} \frac{z}{w}+i \beta \operatorname{Im} \frac{z}{w}\right) \\
& =\alpha^{\prime} \operatorname{Re} \frac{z}{w}+i \beta^{\prime} \operatorname{Im} \frac{z}{w} \\
& =\alpha^{\prime} \operatorname{Re} \frac{f(z)}{f(w)}+i \beta^{\prime} \operatorname{Im} \frac{f(z)}{f(w)} \\
& =f(z) *{ }_{\left(\alpha^{\prime}, \beta^{\prime}\right)} f(w)
\end{aligned}
$$

for all $z, w \in \mathbf{C}$ with $w \neq 0$. Moreover we have

$$
f\left(z *_{(\alpha, \beta)} 0\right)=f(0)=0=f(z) *_{\left(\alpha^{\prime}, \beta^{\prime}\right)} 0=f(z) *\left(\alpha^{\prime}, \beta^{\prime}\right) f(0)
$$

for all $z \in \mathbf{C}$. Consequently we obtain $\Phi(\alpha, \beta) \cong \Phi\left(\alpha^{\prime}, \beta^{\prime}\right)$. 
(b) The case where $\gamma=0$. Note that $\beta=\beta^{\prime}=0$ and $\alpha \neq 0, \alpha^{\prime} \neq 0$. Put

$$
f(z)=\frac{\alpha}{\alpha^{\prime}} z
$$

for each $z \in \mathbf{C}$. Then $f$ is a homeomorphism from $\mathbf{C}$ to itself and

$$
\begin{aligned}
f\left(z *_{\left(\alpha^{\prime}, \beta^{\prime}\right)} w\right) & =\frac{\alpha}{\alpha^{\prime}}\left(z *_{\left(\alpha^{\prime}, \beta^{\prime}\right)} w\right)=\frac{\alpha}{\alpha^{\prime}} \alpha^{\prime} \operatorname{Re} \frac{z}{w}=\alpha \operatorname{Re} \frac{\frac{\alpha}{\alpha^{\prime}} z}{\frac{\alpha}{\alpha^{\prime}} w} \\
& =\alpha \operatorname{Re} \frac{f(z)}{f(w)}=f(z) *(\alpha, \beta)
\end{aligned}
$$

holds for all $z, w \in \mathbf{C}$ with $w \neq 0$. Moreover we have

$$
f\left(z *_{\left(\alpha^{\prime}, \beta^{\prime}\right)} 0\right)=f(0)=0=f(z) *_{(\alpha, \beta)} 0=f(z) *_{(\alpha, \beta)} f(0)
$$

holds for all $z \in \mathbf{C}$. Consequently we obtain $\Phi(\alpha, \beta) \cong \Phi\left(\alpha^{\prime}, \beta^{\prime}\right)$.

(c) The case where $\gamma=\infty$. Note that $\alpha=\alpha^{\prime}=0$ and $\beta \neq 0, \beta^{\prime} \neq 0$. Put

$$
f(z)=\frac{\beta}{\beta^{\prime}} z
$$

for each $z \in \mathbf{C}$. Then $f$ is a homeomorphism from $\mathbf{C}$ to itself and

$$
\begin{aligned}
f\left(z *_{\left(\alpha^{\prime}, \beta^{\prime}\right)} w\right) & =\frac{\beta}{\beta^{\prime}}\left(z *_{\left(\alpha^{\prime}, \beta^{\prime}\right)} w\right)=\frac{\beta}{\beta^{\prime}} \beta^{\prime} i \operatorname{Im} \frac{z}{w}=\beta i \operatorname{Im} \frac{\frac{\beta}{\beta^{\prime}} z}{\frac{\beta}{\beta^{\prime}} w} \\
& =\beta i \operatorname{Im} \frac{f(z)}{f(w)}=f(z) *_{(\alpha, \beta)} f(w)
\end{aligned}
$$

holds for all $z, w \in \mathbf{C}$ with $w \neq 0$. Moreover we have

$$
f\left(z *_{\left(\alpha^{\prime}, \beta^{\prime}\right)} 0\right)=f(0)=0=f(z) *_{(\alpha, \beta)} 0=f(z) *_{(\alpha, \beta)} f(0)
$$

holds for all $z \in \mathbf{C}$. Consequently we obtain $\Phi(\alpha, \beta) \cong \Phi\left(\alpha^{\prime}, \beta^{\prime}\right)$.

IV. Proof of Theorem 4. Let $\alpha, \beta, \alpha^{\prime}, \beta^{\prime} \in \mathbf{C}$ and suppose that $\Phi(\alpha, \beta) \cong$ $\Phi\left(\alpha^{\prime}, \beta^{\prime}\right)$. Let $f$ be a corresponding homeomorphism from $\mathbf{C}$ to itself. Then

$$
f(z *(\alpha, \beta) w)=f(z) *\left(\alpha^{\prime}, \beta^{\prime}\right) f(w)
$$

holds for all $z, w \in \mathbf{C}$. In this case we have

$$
f(0)=0 .
$$

Actually since $f$ is bijective, we can choose a $z_{0} \in \mathbf{C}$ with $f\left(z_{0}\right)=0$. Taking $z=z_{0}$ and $w=0$ in (9), we have $f(0)=f\left(z_{0}\right) *\left(\alpha^{\prime}, \beta^{\prime}\right) f(0)=0 *_{\left(\alpha^{\prime}, \beta^{\prime}\right)} f(0)=0$. 
By (9), (10) and Theorem 2, we have

$$
f\left(\alpha \operatorname{Re} \frac{z}{w}+i \beta \operatorname{Im} \frac{z}{w}\right)=\alpha^{\prime} \operatorname{Re} \frac{f(z)}{f(w)}+i \beta^{\prime} \operatorname{Im} \frac{f(z)}{f(w)}
$$

for all $z, w \in \mathbf{C}$ with $w \neq 0$. Also taking $z=w=1$ in (11), we have

$$
f(\alpha)=\alpha^{\prime} .
$$

(i) Let $\alpha, \beta \in \mathbf{C}$ and suppose that $\Phi(\alpha, \beta) \cong \Phi(0,0)$. If $f$ is a corresponding homeomorphism, then

$$
f(\alpha \operatorname{Re} z+i \beta \operatorname{Im} z)=f\left(z *_{(\alpha, \beta)} 1\right)=f(z) *_{(0,0)} f(1)=0
$$

for all $z \in \mathbf{C}$. Taking $z=1$ in the above equation, we obtain that $f(\alpha)=f(0)$, hence $\alpha=0$ since $f(0)=0$ by (10). Similarly taking $z=i$ in the same equation, we obtain $i \beta=0$, namely, $\beta=0$. Consequently, $\Phi(0,0)$ is not isomorphic to any nontrivial continuous fractional binary operation on $\mathbf{C}$.

(ii) Let $\gamma=0$ or $\infty, \gamma^{\prime} \in \mathbf{C} \backslash\{0\},(\alpha, \beta) \in L_{\gamma}$ and $\left(\alpha^{\prime}, \beta^{\prime}\right) \in L_{\gamma^{\prime}}$ with $\operatorname{Re} \alpha^{\prime} \overline{\beta^{\prime}} \neq 0$. Then we must show that $\Phi(\alpha, \beta) \nsucceq \Phi\left(\alpha^{\prime}, \beta^{\prime}\right)$.

(ii-a) The case where $\gamma=0$. Note that $\beta=0$ and $\alpha \neq 0$. Assume that $\Phi(\alpha, \beta) \cong$ $\Phi\left(\alpha^{\prime}, \beta^{\prime}\right)$. By Theorem 3,

$$
\Phi\left(\alpha^{\prime}, \beta^{\prime}\right) \cong \Phi(\alpha, \beta)=\Phi(\alpha, 0) \cong \Phi(1,0) .
$$

Then $\Phi\left(\alpha^{\prime}, \beta^{\prime}\right) \cong \Phi(1,0)$, so let $f$ be its corresponding homeomorphism. Then we have from (11) that

$$
f\left(\alpha^{\prime} \operatorname{Re} \frac{z}{w}+i \beta^{\prime} \operatorname{Im} \frac{z}{w}\right)=\operatorname{Re} \frac{f(z)}{f(w)}
$$

holds for all $z, w \in \mathbf{C}$ with $w \neq 0$. Taking $w=1$ in the above equation, we obtain that

$$
f\left(\alpha^{\prime} \operatorname{Re} z+i \beta^{\prime} \operatorname{Im} z\right)=\operatorname{Re} \frac{f(z)}{f(1)} \in \mathbf{R}
$$

for all $z \in \mathbf{C}$. Since $\operatorname{Re} \alpha^{\prime} \overline{\beta^{\prime}} \neq 0$ by hypothesis, we can easily see that

$$
\left\{w \in \mathbf{C}: w=\alpha^{\prime} \operatorname{Re} z+i \beta^{\prime} \operatorname{Im} z, z \in \mathbf{C}\right\}=\mathbf{C} .
$$

Therefore we have from (13) that $f(\mathbf{C})=\mathbf{R}$, a contradiction. Consequently, $\Phi(\alpha, \beta) \nsucceq$ $\Phi\left(\alpha^{\prime}, \beta^{\prime}\right)$.

(ii-b) The case where $\gamma=\infty$. Note that $\alpha=0$ and $\beta \neq 0$. Assume that $\Phi(\alpha, \beta) \cong$ $\Phi\left(\alpha^{\prime}, \beta^{\prime}\right)$. By Theorem 3,

$$
\Phi\left(\alpha^{\prime}, \beta^{\prime}\right) \cong \Phi(\alpha, \beta)=\Phi(0, \beta) \cong \Phi(0,1) .
$$


Then $\Phi\left(\alpha^{\prime}, \beta^{\prime}\right) \cong \Phi(0,1)$, so let $g$ be its corresponding homeomorphism. Then we have from (11) that

$$
g\left(\alpha^{\prime} \operatorname{Re} \frac{z}{w}+i \beta^{\prime} \operatorname{Im} \frac{z}{w}\right)=i \operatorname{Im} \frac{g(z)}{g(w)}
$$

holds for all $z, w \in \mathbf{C}$ with $w \neq 0$. Taking $w=1$ in the above equation, we obtain that

$$
g\left(\alpha^{\prime} \operatorname{Re} z+i \beta^{\prime} \operatorname{Im} z\right)=i \operatorname{Im} \frac{g(z)}{g(1)} \in i \mathbf{R}
$$

for all $z \in \mathbf{C}$. Since $\operatorname{Re} \alpha^{\prime} \overline{\beta^{\prime}} \neq 0$ by hypothesis, it follows from (13) that $g(\mathbf{C})=i \mathbf{R}$, a contradiction. Consequently, $\Phi(\alpha, \beta) \varsubsetneqq \Phi\left(\alpha^{\prime}, \beta^{\prime}\right)$.

(iii) Let $(\alpha, \beta) \in L_{0}$ and $\left(\alpha^{\prime}, \beta^{\prime}\right) \in L_{\infty}$. Assume that $\Phi(\alpha, \beta) \cong \Phi\left(\alpha^{\prime}, \beta^{\prime}\right)$. Since $\alpha \neq 0, \beta=0, \alpha^{\prime}=0$ and $\beta^{\prime} \neq 0$, it follows from Theorem 3 that

$$
\Phi(0,1) \cong \Phi\left(0, \beta^{\prime}\right)=\left(\Phi\left(\alpha^{\prime}, \beta^{\prime}\right) \cong \Phi(\alpha, \beta)=\Phi(\alpha, 0) \cong \Phi(1,0) .\right.
$$

Then $\Phi(0,1) \cong \Phi(1,0)$, so let $f$ be its corresponding homeomorphism. Then we have from (11) that

$$
f\left(i \operatorname{Im} \frac{z}{w}\right)=\operatorname{Re} \frac{f(z)}{f(w)}
$$

holds for all $z, w \in \mathbf{C}$ with $w \neq 0$. Taking $z=w=1$ in the above equation, we obtain that

$$
f(0)=f(i \operatorname{Im} 1)=\operatorname{Re} \frac{f(1)}{f(1)}=\operatorname{Re} 1=1
$$

Since $f(0)=0$ by (10), it follows that $0=1$, a contradiction. Consequently, $\Phi(\alpha, \beta) \nsucceq$ $\Phi\left(\alpha^{\prime}, \beta^{\prime}\right)$.

(iv) Let $\alpha, \beta \in C \backslash\{0\}$ and suppose that $\Phi(\alpha, \beta) \cong \Phi(1,1)$. Let $f$ be a corresponding homeomorphism. Then $f(\alpha)=1$ by (12). Also we have from (11) that

$$
f\left(\alpha \operatorname{Re} \frac{z}{w}+i \beta \operatorname{Im} \frac{z}{w}\right)=\frac{f(z)}{f(w)}
$$

for all $z, w \in \mathbf{C}$ with $w \neq 0$. Taking $w=\alpha$ in the above equation, we obtain

$$
f\left(\alpha \operatorname{Re} \frac{z}{\alpha}+i \beta \operatorname{Im} \frac{z}{\alpha}\right)=\frac{f(z)}{f(\alpha)}=f(z)
$$

for all $z \in \mathbf{C}$. Since $f$ is injective, it follows that

$$
\alpha \operatorname{Re} \frac{z}{\alpha}+i \beta \operatorname{Im} \frac{z}{\alpha}=z
$$

holds for all $z \in \mathbf{C}$. Taking $z=i \alpha$ in the above equation, we obtain $i \beta=i \alpha$, hence $\alpha=\beta$. 
The converse follows immediately from Theorem 3 .

IV. Proof of TheOREM 5. Let $*$ be a nontrivial homogeneous cancellative binary operation on K. Put

$$
\varphi(b)=1 * b
$$

for each $b \in \mathbf{K}$. Then we have $a * b=a \varphi(b)$ for all $a, b \in \mathbf{K}$. Then $*$ must be isomorphic to Saitoh's fraction on $\mathbf{K}$ as observed in the proof of Theorem 1.

ACKNOWLEDGMENTS. The authors would like to thank the referee for his helpful comments. Also we thank Professor emeritus Saburou Saitoh for his encouragement in preparing the paper and Professor emeritus Jun Tomiyama for his useful comments.

This research was partially supported by Grant-in-Aid for Scientific Research, Japan Society for the Promotion of Science, No. 25400120.

\section{References}

[ 1 ] L. P. CASTRO and S. SAITOH, Fractional functions and their representations, Complex Analysis and Operator Theory 7 (2013), 1049-1063. DOI: 10.1007/s11785-011-0154-1.

[2 ] S. SAITOH, A natural interpretation on $100 / 0=0$ and $0 / 0=0$, and an open question, preprint.

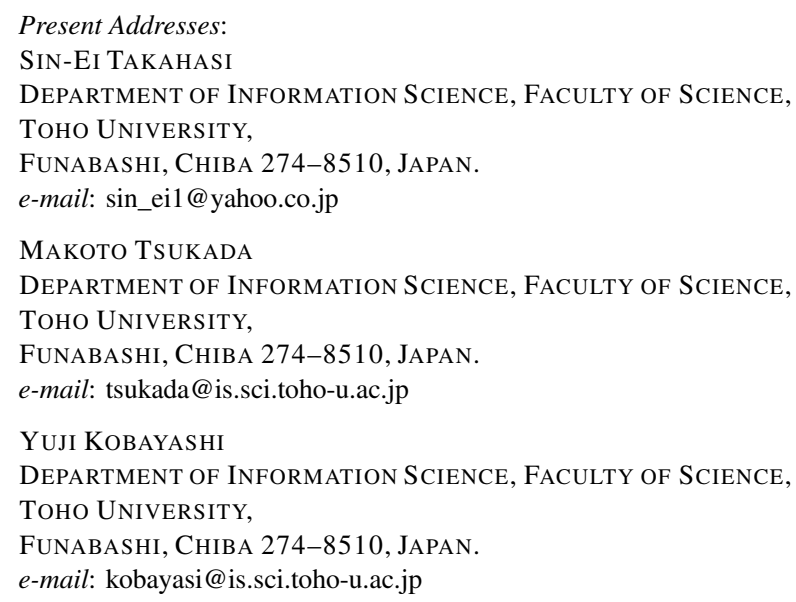

